\title{
Multimodal Imaging of Spontaneously Shifting Primary Vitreoretinal Lymphoma
}

\author{
Dimosthenis Mantopoulos Colleen M. Cebulla
}

Havener Eye Institute, Department of Ophthalmology and Visual Science, The Ohio State University Wexner Medical Center, Columbus, Ohio, USA

\section{Key Words}

Primary vitreoretinal lymphoma - Spectral domain optical coherence tomography . Spontaneous regression $\cdot$ Immune surveillance

\begin{abstract}
Purpose: To correlate spectral domain optical coherence tomography (SD-OCT) and photographic imaging before and after spontaneous regression of primary vitreoretinal lymphoma (PVRL) lesions. Procedures: We report the case of a 60-year-old female. Results: The patient presented with bilateral creamy deposits under the retina and retinal pigment epithelium (RPE), and lesions were visible along Bruch's membrane with SD-OCT and suspicious for PVRL. Systemic workup revealed nonspecific areas of enhancement on neuroimaging. The patient was largely asymptomatic and the decision was made to observe her. Three months later, a new lesion pattern had developed. The color fundus photographs and SD-OCT demonstrated spontaneous regression of the largest sub-RPE lesion, leaving areas of RPE atrophy, while a new larger sub-RPE lesion had formed in the other eye. Vitreous biopsy showed lymphocytes and no malignant cells, while sub-RPE biopsy of the newly formed lesion revealed highly atypical cells positive for CD19 and CD20. Conclusions: Multimodal imaging documents that PVRL lesion regression and early RPE changes can develop within a 3-month period. Immune control is an important factor in lesion regression in the eye.

(C) 2015 S. Karger AG, Basel
\end{abstract}

\section{Introduction}

Primary vitreoretinal lymphoma (PVRL) is a rare subset of primary central nervous system (CNS) lymphoma [1, 2]. While malignancies rarely spontaneously regress, subretinal PVRL lesions have been previously reported to migrate or regress without treatment [3]. 


\section{Ocular Oncology and Pathology}

\begin{tabular}{l|l}
\hline Ocul Oncol Pathol 2015;1:237-240 \\
\hline DOI: 10.1159/000374121 & $\begin{array}{l}\text { @ 2015 S. Karger AG, Basel } \\
\text { www.karger.com/oop }\end{array}$ \\
\hline
\end{tabular}

Mantopoulos and Cebulla: Multimodal Imaging of Spontaneously Shifting Primary Vitreoretinal Lymphoma
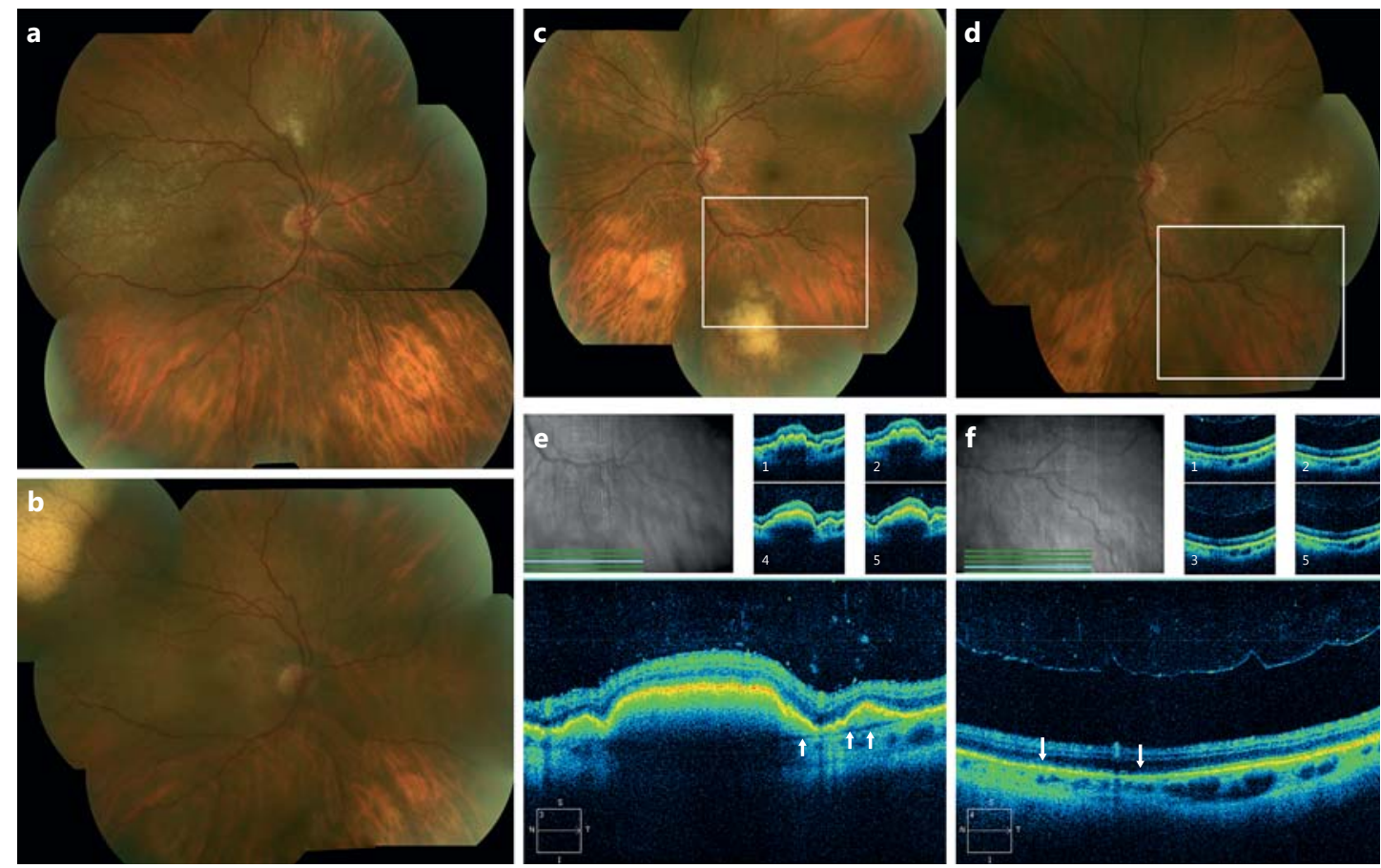

Fig. 1. Montage fundus photographs of the OD and OS at presentation (a, c). Note the creamy sub-RPE infiltrates in the macula, temporally, and superiorly in the OD (a) and the coalesced, fluffy sub-RPE infiltrates inferior to the inferior arcade and the discrete infiltrates superonasally in the OS (c). Three months later, many of these lesions resolved without any treatment (b OD, d OS), while a new sub-RPE lesion appeared at 10 o'clock in the OD (b) and temporal to the macula in the OS (d). SD-OCT in the area of the inferior lesion on presentation (e) showed RPE elevation secondary to prominent sub-RPE material accumulation along Bruch's membrane (arrows) and some small subretinal material accumulation, with blocked visualization of much of the choriocapillaris in the OS. Three months later, the area where the lesion regressed showed RPE atrophy and irregularity (arrows) and improved visualization of the choriocapillaris on SD-OCT (f).

However, there is little documentation of this observation in the literature with modern imaging techniques. Here, we correlate the photographic and spectral domain optical coherence tomography (SD-OCT) findings in a case of spontaneous lesion regression in a treatment-naïve patient with PVRL.

\section{Case Report}

The patient is a 60-year old female with a past medical history significant for hypothyroidism and type 2 diabetes. Her cancer family history was positive for lymphoma and prostate cancer in her father. She had a 1-year history of intermittent scotomata and decreased vision in OU and a 3-week history of photophobia and metamorphopsia in OU resistant to topical prednisolone that she received from an outside clinic. The review of systems was otherwise unremarkable.

On presentation to our department, her visual acuity was 20/30 in the OD, 20/20 in the OS with quiet anterior segments and vitreous OU and multiple subretinal/subretinal pigment epithelium (RPE) creamy deposits in OU (fig. 1a, c). Fundus autofluorescence pictures showed a mixed pattern of increased and decreased autofluorescence (fig. 2a), while fluorescein angiography demonstrated early blockage in the subretinal material followed by late mild hyperfluorescence (fig. 2b, c). The SD-OCT showed accumulation of predominantly sub-RPE material along Bruch's membrane (fig. 1e). 
Ocular Oncology and Pathology

Mantopoulos and Cebulla: Multimodal Imaging of Spontaneously Shifting Primary Vitreoretinal Lymphoma
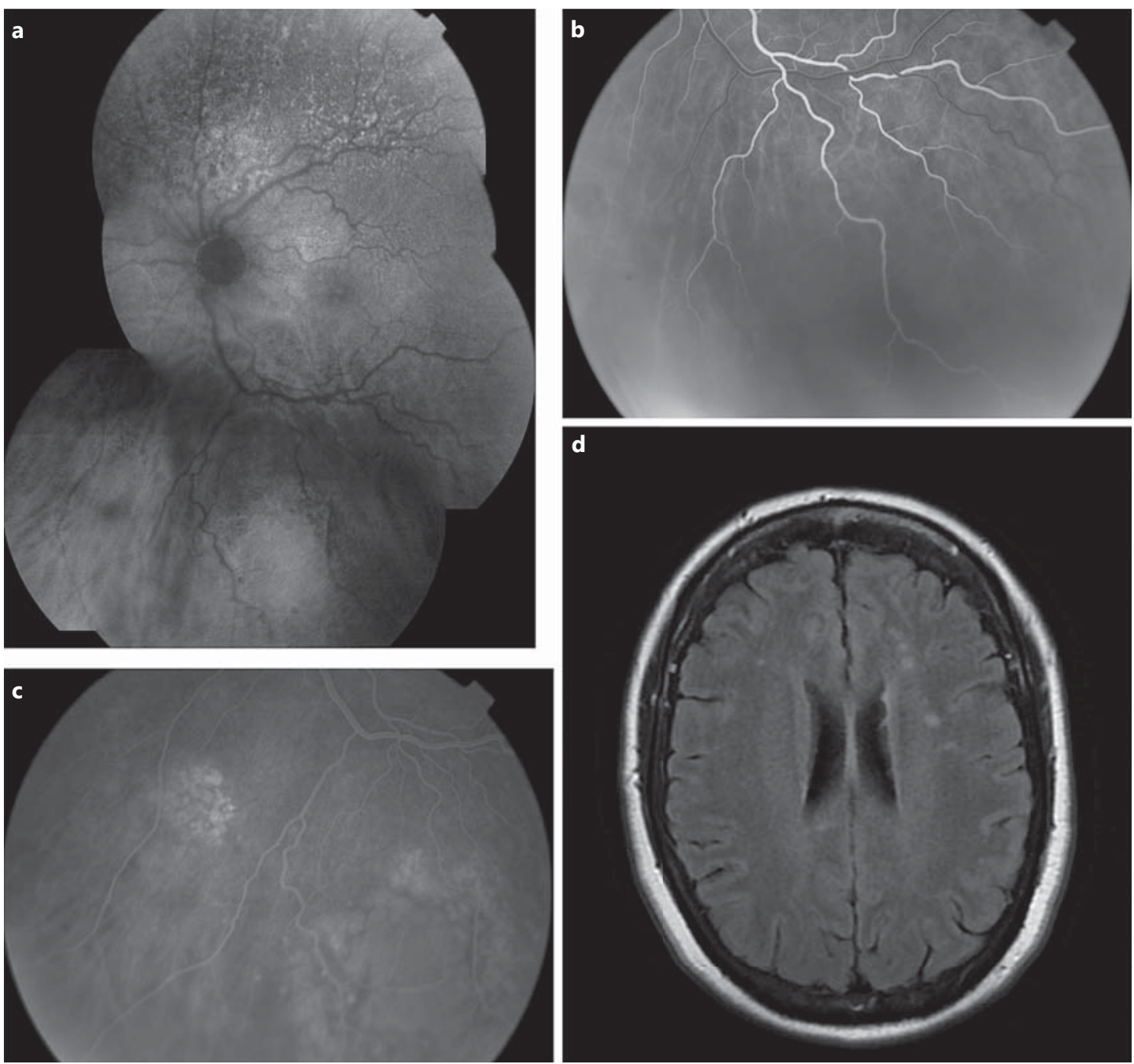

(C) 2015 S. Karger AG, Base www.karger.com/oop 
Vitrectomy and sub-RPE biopsy of the largest lesion were performed in the OD. Cytology of the vitreous showed lymphocytes and an eosinophil. Flow cytometry and cytology of the sub-RPE biopsy were negative for CD8+ T cells or NK cells and positive for a dominant population of highly atypical B cells co-expressing CD19 and CD20, consistent with B-cell lymphoproliferative disorder. Thus, systemic treatment with rituximab and methotrexate was initiated by neuro-oncology given the mild CNS findings on MRI. The patient's lesions regressed well with systemic treatment.

\section{Discussion}

Spontaneous resolution of the primary neoplastic lesion is an unusual phenomenon in any malignancy, but this observation has been made for PVRL and other non-Hodgkin's lymphoma types $[3,4]$. The color fundus photographs and SD-OCT images of this report provide documentation that PVRL lesions can regress without treatment, leaving an area of RPE atrophy. The exact mechanism or clinical significance of these lesion changes is hypothesized to be due to tumor control by the host immune system, primarily by CD8+ T cells [3, 4] as well as NK cells [5]. In this case, the patient's vitreous biopsy showed a predominant population of non-neoplastic lymphocytes, providing support of immune surveillance, while the sub-RPE biopsy of the newly formed lesion did not. Further elucidation of these mechanisms of immunologic tumor control and migration is valuable since it could open new therapeutic approaches against the malignant process.

\section{Acknowledgments}

This article was supported by the National Eye Institute of the National Institutes of Health under Award No. K08EY022672. Additional funds were provided by the Ohio Lions Eye Research Foundation, Fund \#313310 in Ophthalmology, and the Patti Blow Fund.

\section{Disclosure Statement}

The authors have no conflicts of interest to disclose. The content of this paper is solely the responsibility of the authors and does not necessarily represent the official views of these institutions.

\section{References}

1 Davis JL: Intraocular lymphoma: a clinical perspective. Eye 2013;27:153-162.

2 Chan CC, Rubenstein JL, Coupland SE, Davis JL, Harbour JW, Johnston PB, Cassoux N, Touitou V, Smith JR, Batchelor TT, Pulido JS: Primary vitreoretinal lymphoma: a report from an International Primary Central Nervous System Lymphoma Collaborative Group symposium. Oncologist 2011;16:1589-1599.

3 Kase S, Namba K, Jin XH, Kubota KC, Ishida S: Spontaneous regression of intraocular lymphoma. Ophthalmology 2012;119:1083-1084.

4 Iihara K, Yamaguchi K, Nishimura Y, Iwasaki T, Suzuki K, Hirabayashi Y: Spontaneous regression of malignant lymphoma of the breast. Pathol Int 2004;54:537-542.

5 Ono K: Clinical significance of natural killing activity in patients with advanced lymphoma. J Clin Immunol 1998;18:132-141. 